\title{
A novel protective effect of erythropoietin in the infarcted heart
}

\author{
Cyrus J. Parsa, ${ }^{1}$ Akio Matsumoto,${ }^{2}$ Jihee Kim, ${ }^{1}$ Ryan U. Riel, ${ }^{1}$ Laura S. Pascal, ${ }^{1}$ \\ G. Brant Walton, ${ }^{1}$ Richard B. Thompson, ${ }^{1}$ Jason A. Petrofski, ${ }^{1}$ Brian H. Annex, ${ }^{3}$ \\ Jonathan S. Stamler, ${ }^{2,3,4}$ and Walter J. Koch ${ }^{1}$ \\ ${ }^{1}$ Department of Surgery, \\ ${ }^{2}$ Howard Hughes Medical Institute, \\ ${ }^{3}$ Department of Medicine (Cardiology), and \\ ${ }^{4}$ Department of Medicine (Pulmonary), Duke University Medical Center, Durham, North Carolina, USA
}

\begin{abstract}
Erythropoietin (EPO) has been shown to protect neurons from ischemic stroke, but can also increase thrombotic events and mortality rates in patients with ischemic heart disease. We reasoned that benefits of EPO might be offset by increases in hematocrit and evaluated the direct effects of EPO in the ischemic heart. We show that preconditioning with EPO protects H9c2 myoblasts in vitro and cardiomyocytes in vivo against ischemic injury. EPO treatment leads to significantly improved cardiac function following myocardial infarction. This protection is associated with mitigation of myocyte apoptosis, translating into more viable myocardium and less ventricular dysfunction. EPO-mediated myocyte survival appears to involve Akt activation. Importantly, cardioprotective effects of EPO were seen without an increase in hematocrit (eliminating oxygen delivery as an etiologic factor in myocyte survival and function), demonstrating that EPO can directly protect the ischemic and infarcted heart.
\end{abstract}

J. Clin. Invest. 112:999-1007 (2003). doi:10.1172/JCI200318200.

\begin{abstract}
Introduction
Cardiovascular disease remains the number one cause of mortality in the United States and is fast becoming the number one health concern worldwide. Preservation of myocardium by eliminating ischemia acutely following myocardial infarction (MI) is a major therapeutic objective of drugs such as thrombolytics and platelet antagonists. An alternative approach that may have clinical utility is to protect the post-MI heart against programmed cell death or apoptosis induced by ischemic conditions (1). Protection of cardiomyocytes from ischemia/hypoxia-induced cell death would increase viable myocardium, providing acute and chronic benefits after MI.

The hematopoietic cytokine erythropoietin (EPO) and its receptor (EPO-R) have been shown to be present in tissues outside the blood, including the heart (2-4), suggesting potential roles of EPO signaling beyond hematopoiesis and the treatment of anemia $(5,6)$. EPO
\end{abstract}

Received for publication February 24, 2003, and accepted in revised form July 29, 2003.

Address correspondence to: Walter J. Koch, Center for Translational Medicine, Jefferson Medical College, 1025 Walnut, Room 822, Philadelphia, Pennsylvania 19107, USA

Phone: 215-955-9982; Fax: 215-955-1690;

E-mail: walter.koch@jefferson.edu.

Conflict of interest: The authors have declared that no conflict of interest exists.

Nonstandard abbreviations used: myocardial infarction (MI); erythropoietin (EPO); erythropoietin receptor (EPO-R);

Janus-associated kinase-2 (Jak2); trichloroacetic acid (TCA); left circumflex coronary artery (LCx); reactive oxygen species (ROS); left ventricle (LV); triphenyltetrazolium chloride (TTC);

$\beta$-adrenergic receptor ( $\beta \mathrm{AR}) ; \mathrm{LV}$ end-diastolic pressure (LVEDP). is produced by the kidney in response to hypoxia and stimulates erythroid progenitor cells to increase the number of mature red blood cells, thereby increasing $\mathrm{O}_{2}$ carrying capacity $(5,7)$. The effect of EPO on oxygen delivery is, however, not straightforward because increases in hematocrit may titrate $\mathrm{NO}$ and thereby impair tissue blood flow (8-10). Transgenic animals overexpressing EPO die rapidly from cardiovascular dysfunction when administered a NO synthase inhibitor (8). Furthermore, EPO can elevate blood pressure and the incidence of thrombosis (10). Finally, increases in hematocrit have been linked to excess mortality rates in patients with ischemic heart disease $(11,12)$.

In contrast to these negative events associated with $\mathrm{EPO}$ and hematopoiesis, accumulating evidence indicates that EPO is a cellular survival factor in neurons $(2,3,13,14)$. Examples of the neuronal protection by EPO include cerebral infarct reduction after middle cerebral artery occlusion in a rodent model (2). Administration of $5,000 \mathrm{U} / \mathrm{kg}$ of EPO 24 hours before, concomitantly, or even up to 9 hours after reversible middle cerebral artery occlusion attenuated the volume of cerebral infarction as much as $75 \%$ in rats (3). In the retina, EPO upregulation (in response to hypoxia) or exogenous administration prevents light-induced retinal degeneration (13). Other studies show that EPO and subsequent EPO-R signaling provides cellular protection by inhibiting apoptosis involving specific protein kinase cascades, including the stress-responsive Janus-associated kinase-2 (Jak2) $(15,16)$, PI3K/Akt, and ras-MAP kinase pathways $(16,17)$. Downstream mediators may also include $\mathrm{NO}$, which is antiapoptotic (18). 
Given the data concerning neuronal protection by EPO following hypoxic/ischemic injury and the fact that the EPO-R is expressed in the heart (4), we posited that EPO may exert cardioprotective effects. We reasoned that salutary actions in the heart might be counterbalanced in vivo by increases in hematocrit and so have addressed whether EPO can protect (a) isolated cardiac myocytes in vitro from $\mathrm{H}_{2} \mathrm{O}_{2}$ treatment, a form of oxidative stress-simulating ischemic tissue injury (19), as well as cells subjected to hypoxia, and (b) ischemic hearts in vivo under conditions where EPO does not increase hematocrit. Our results show that the erythroid cytokine EPO activates cell survival pathways resulting in inhibition of myocyte apoptosis in vitro and also in the penumbra of the ischemic heart, resulting in increased viable myocardium (decreased infarct size) and enhanced post-MI global contractile function. Thus, acute administration of EPO may reduce myocardial damage following ischemic injury and represent a novel therapy for acute coronary syndromes.

\section{Methods}

Materials. The H9c2 myoblast cell line derived from embryonic rat heart (20) obtained from American Type Culture Collection (Manassas, Virginia, USA) was used for all in vitro experiments. Cells were maintained in high-glucose DMEM supplemented with 10\% FBS, penicillin $\mathrm{G}(100 \mathrm{U} / \mathrm{ml})$, and streptomycin $(100 \mu \mathrm{g} / \mathrm{ml})$ without tubular structure formation, under $5 \% \mathrm{CO}_{2}$ at $37^{\circ} \mathrm{C}$. The MAPK kinase (MEK) inhibitor (PD98059) and PI3K inhibitor (wortmannin) were obtained from Calbiochem (San Diego, California, USA). Isoproterenol (ISO), heparin, and PDGF were purchased from Sigma-Aldrich (St. Louis, Missouri, USA). Polyclonal anti-phospho-STAT3 and anti-STAT3 used for immunoblotting of STAT3 were from Cell Signaling Technology (Beverly, Massachusetts, USA). Polyclonal anti-phospho Jak1 and anti-Jak1 were from Biosource International (Camarillo, California, USA). Polyclonal anti-phosphorylated ERK, polyclonal anti-phosphorylated Akt, and polyclonal anti-Akt from Cell Signaling Technology and polyclonal anti-ERK2 from Santa Cruz Biotechnology (Santa Cruz, California, USA) were used for ERK and Akt activation assay. $\left[{ }^{3} \mathrm{H}\right]$-thymidine was purchased from Amersham Biosciences (Piscataway, New Jersey, USA).

Western blot analysis. Western blot analysis was performed for ERK, Akt, Jak1, and STAT3 activation using specific antiphosphorylated Ab's. H9c2 cells were serum starved in DMEM with $0.1 \%$ BSA for 24 hours. Serumstarved cells in six-well plates were stimulated with 4 $\mathrm{U} / \mathrm{ml}$ of EPO for the indicated time at $37^{\circ} \mathrm{C}$. After stimulation, monolayers were washed once with ice-cold PBS and lysed in buffer containing $50 \mathrm{mM}$ HEPES, $\mathrm{pH}$ 7.5, $150 \mathrm{mM} \mathrm{NaCl}$, 1\% Triton X-100, 1 mM EDTA, 10\% glycerol, $10 \mathrm{mM}$ sodium pyrophosphate, $2 \mathrm{mM}$ sodium orthovanadate, $10 \mathrm{mM}$ sodium fluoride, $1 \mathrm{mM}$ phenylmethylsulfonyl fluoride, and $10 \mu \mathrm{g} / \mathrm{ml}$ aprotinin. After lysis, the same amount of proteins was applied for
4-20\% polyacrylamide/Tris-glycine gel. Phosphorylated protein was then visualized by enzyme-linked chemiluminescence (Amersham Biosciences) and quantified by scanning laser densitometry, normalizing to total amounts of the corresponding proteins.

DNA synthesis. DNA synthesis was determined as incorporation of $\left[{ }^{3} \mathrm{H}\right]$-thymidine into trichloroacetic acid-insoluble (TCA-insoluble) material. Plates $(n=6)$ were seeded at an initial density of $2 \times 10^{4}$ cells/well in 12 wells and grown to subconfluence. Cells were rendered quiescent by 48 hours in serum-free DMEM supplemented with $0.1 \%$ BSA and stimulated by the addition of fresh medium containing the agonists. $\left[{ }^{3} \mathrm{H}\right]$-thymidine $(1 \mu \mathrm{Ci} / \mathrm{ml})$ was added to the medium at the time of stimulation. Cells were washed twice with PBS and three times with $0.5 \%$ TCA, and then cells were lysed with $1 \mathrm{~N} \mathrm{NaOH}$. Extent of cell growth was determined by counting incorporation of $\left[{ }^{3} \mathrm{H}\right]$-thymidine into nascent DNA strands at 48 hours.

Apoptosis quantification after $\mathrm{H}_{2} \mathrm{O}_{2}$ treatment and bypoxia. $\mathrm{H} 9 \mathrm{c} 2$ cells were plated at $3 \times 10^{5} /$ well in six-well tissue culture plates and pretreated with EPO (0.4 or 10 $\mathrm{U} / \mathrm{ml}$ ) or vehicle for 24 hours prior to beginning the $\mathrm{H}_{2} \mathrm{O}_{2}(200 \mu \mathrm{M})$ treatment in DMEM without glucose and FBS. Following $\mathrm{H}_{2} \mathrm{O}_{2}$ treatment for 22 hours, adherent cells were fixed with $4 \%$ paraformaldehyde, and nuclei were stained with Hoechst 33258 dye to visualize morphological changes by fluorescence microscopy. Alternatively, similar $\mathrm{H} 9 \mathrm{c} 2$ cell densities were plated into 12 -well plates at $75 \%$ confluence. Experimental wells received EPO (8 U/ml), PD98059 (5 $\mu \mathrm{g} / \mathrm{ml})$, wortmannin $(500 \mathrm{nM})$, and combinations thereof in the culture media. All plates were subjected to 12 hours of anoxia $\left(95 \% \mathrm{~N}_{2}, 5 \% \mathrm{CO}_{2}\right)$ and subsequently fixed and stained as detailed above. Apoptotic cell death was determined by bright staining on condensed nuclei or discrete fragmented nuclei (21).

Induction of MI. Animals used in this in vivo study were adult male New Zealand White rabbits $(2.5-3.5 \mathrm{~kg})$, and all procedures were performed in accordance with the regulations adopted by the NIH and approved by the Animal Care and Use Committee of Duke University. MI was induced in anesthetized rabbits by a left thoracotomy and ligation of a branch of the left circumflex coronary artery (LCx) as described previously (22). Rabbits were sedated with a mixture of ketamine $(30 \mathrm{mg} / \mathrm{kg})$ and acepromazine $(0.05 \mathrm{mg} / \mathrm{kg})$, intubated, and mechanically ventilated. Inhalation anesthesia (isoflurane) was titrated to maintain the absence of pedal and corneal reflexes. Animals were preoperatively randomized to receive a single dose of either EPO (5,000 U/kg; Amgen Inc., Thousand Oaks, California, USA) $(n=12)$ or normal saline $(3 \mathrm{ml})(n=11)$ by the animal technician at the time of MI. Sham animals $(n=5)$ underwent thoracotomies in which the LCx was circled with a prolene suture, but was not occluded. Rabbits were sacrificed on postoperative day 4 , and their hearts were harvested for infarction quantification. Infarct size, as a percentage of the left ventricle (LV) and septal 
mass, was quantified using triphenyltetrazolium chloride (TTC) staining as described previously (23). Fixed heart sections were placed on a camera mount to standardize camera-to-specimen distance, and digital photographs were taken. Using enlarged projections, the boundary of the stained areas on each slice was traced in a blinded fashion, and Adobe Photoshop 6.0 (Adobe Systems Inc., San Jose, California, USA) was used to quantify infarcted versus normal myocardium.

Assessment of area at risk. Rabbits were anesthetized and underwent a thoracotomy as described above. Rabbits were anticoagulated with intravenous heparin (70 $\mathrm{U} / \mathrm{kg}$ ), followed by 30 minutes of LCx occlusion. In a randomized fashion, rabbits received $\mathrm{EPO}(5,000 \mathrm{U} / \mathrm{kg})$ $(n=5)$ or an equivalent volume of normal saline $(n=5)$ following LCx occlusion via a peripheral ear vein. At the start of reperfusion, a marking stitch was left at the site of occlusion, and on day 3 the animals were sacrificed and their hearts harvested. The area of the LV at risk for ischemic injury was stained as described $(23,24)$ using TTC staining, followed by counterstaining with pthalo blue after occlusion at the previous ligation site using the marking stitch. The heart was then dissected, cut into five or six 2-mm-thick transverse slices, fixed, and photographed as described (23). Total weights of area at risk and area of necrosis were then blindly calculated and expressed as percentage of total LV weight.

TUNEL. In the subset of animals chosen for in vivo TUNEL assessment, animals were pretreated with $1,000 \mathrm{U} / \mathrm{kg}$ EPO $(n=4)$ or normal saline $(n=4) 24$ hours prior to LCx ligation and survived for 6 hours. Histologic heart samples were frozen in cryomolds, and TUNEL labeling was performed on $5-\mu \mathrm{m}$ sections per the manufacturer's protocol (In Situ Cell Death Detection kit; Roche Diagnostics, Indianapolis, Indiana, USA). A notable exception was that the cell membrane-permeablization step was extended to $15 \mathrm{~min}$ utes. Digital photographs were taken under fluorescence microscopy at $\times 200$ magnification. Twenty-five random high-power fields from each heart sample were chosen and blindly quantified.

In vivo kinase activity. For assessment of $\mathrm{Akt}$ and ERK activation, normal rabbits received either 1,000 $\mathrm{U} / \mathrm{kg}(n=4)$ or saline (control, $n=4)$ intravenously and were sacrificed 12 hours later. LV samples were then homogenized in the buffer described above for the cell experiments, and activated Akt and ERK were assessed by protein immunoblotting using Ab's raised against the phosphorylated kinases and quantified as described above.

In vivo hemodynamic measurements. All animals underwent hemodynamic assessment 24 hours prior to MI or sham operation, and a repeat assessment was done on postoperative day 3 , as we have described previously (22). $\mathrm{LV}$ pressure and heart rate were measured by a 2.5 French micromanometer (Millar Instruments Inc., Houston, Texas, USA) advanced into the LV cavity, and data were recorded on a PowerLab System (ADInstruments Pty Ltd., Mountain View, California, USA) as described (22).
Hematocrit measurements. Blood samples were obtained from a peripheral ear vein of sedated rabbits at serial time points: day $0,1,3$, and 4 . Samples were analyzed using a blood gas/electrolyte analyzer (Model 5500; Instrumentation Laboratory, Ann Arbor, Michigan, USA).

Statistical analysis. Hemodynamic data was analyzed using two-way ANOVA (defined by treatment group and ISO dose) performed with the SAS (SAS Institute Inc., Cary, North Carolina) statistical software program. Densitometry data, apoptotic nuclei data, hematocrit data, infarction, and area at risk data are presented as mean plus or minus SEM. Comparisons between two groups were made using Student's $t$ test. For all analyses, $P$ values less than 0.05 were considered significant.

\section{Results}

EPO signaling and proliferation in H9c2 myoblasts. EPO is known to stimulate three common cell survival pathways, including PI3K/Akt, ERK1/2 MAPK, and Jak-STAT in hematopoietic (25-27) and endothelial (28) cell lines. ERK and Akt activation (via phosphorylation) were assessed in $\mathrm{H} 9 \mathrm{c} 2$ cardiomyoblasts after EPO addition and quantified by Western blot analysis and densitometry at serial time points (1-60 minutes) to determine signaling intermediates (Figure 1, a and b). EPO induced significant Akt activation within 15 minutes and lasted up to 60 minutes (1.5-fold \pm 0.1 -fold over control; $P=0.008)$. ERK was activated 2.96 -fold \pm 0.4 -fold after 60 minutes versus control $(P=0.0016)$. Significant Jak1 phosphorylation occurs at 15 minutes ( 1.39 -fold \pm 0.18 -fold over control, $P=0.027$ ) and continues to serially increase up to 60 minutes $(2.41$-fold \pm 0.54 -fold, $P=0.014$ ) (Figure 1c). One of the downstream intermediates, STAT3, indicates peak activation at 10 minutes (2.07-fold \pm 0.16 -fold versus control, $P=0.0004$ ) with progressive mitigation of phosphorylation over serial time points (Figure 1d). Akin to its effect in erythroblasts $(5,7)$, EPO could stimulate proliferation in cardiac myoblasts. H9c2 cellular proliferation, as measured by $\left[{ }^{3} \mathrm{H}\right]$-thymidine incorporation, mildly increased $9.3 \% \pm 1.4 \%$ using EPO $(4.0 \mathrm{U} / \mathrm{ml})$ compared with control $(P<0.05)$. As a reference, the positive mitogen PDGF induced $12.2 \% \pm 2.1 \%$ proliferation in H9c2 cells $(P<0.05$ versus control).

EPO's antiapoptotic effect in H9c2 myoblasts. The H9c2 cell line is known to undergo apoptotic cell death with $\mathrm{H}_{2} \mathrm{O}_{2}$ treatment (29) and was thus used to assess cardiovascular cell protective properties of EPO. Tissue ischemia is associated with respiratory uncoupling and reactive oxygen species (ROS) production $(30,31)$, and thus, $\mathrm{H}_{2} \mathrm{O}_{2}$ represents an in vitro model of ischemic injury due to oxidative stress (19). H9c2 cells were treated with $200 \mu \mathrm{M} \mathrm{H}_{2} \mathrm{O}_{2}$ for 22 hours to see the effect of pretreatment with EPO to protect cells from apoptosis. H9c2 cells pretreated with EPO exhibited increased survival when compared with cells that did not receive EPO treatment (Figure 2). As shown in Figure $2 \mathrm{a}$, this protection appears to be due to inhibition 


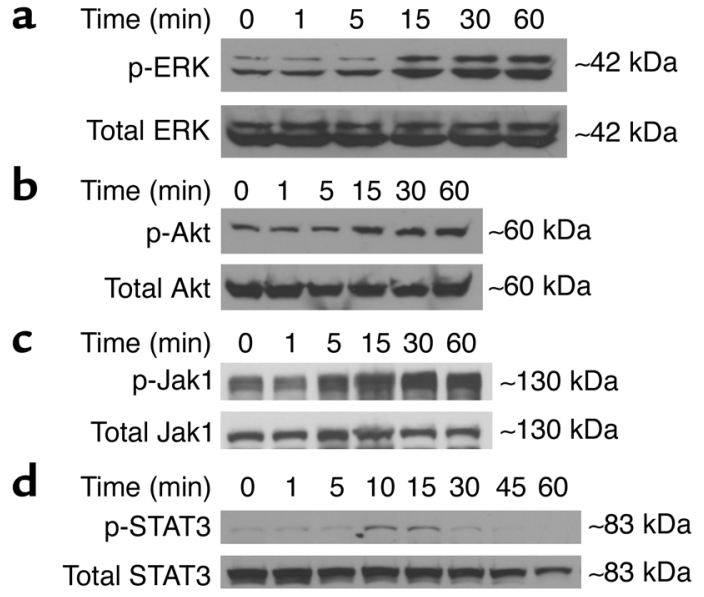

\section{Figure 1}

EPO-mediated activation of critical intracellular kinase cascades including cell survival pathways. Shown are representative Western blots for $\operatorname{ERK}(\mathbf{a}), \operatorname{Akt}(\mathbf{b})$, Jak1 (c), and STAT3 (d) over serial time points. Activated $(\mathrm{p}-)$ signaling intermediates were assessed using antiphospho Ab's, and activated forms were normalized to total protein content of the corresponding molecule (lower panel of all blots). Data are representative of $n=3-4$ experiments.

of apoptosis; after exposure to $\mathrm{H}_{2} \mathrm{O}_{2}$, cells pretreated with EPO (either 0.4 or $10 \mathrm{U} / \mathrm{ml}$ ) exhibited a significant $(\sim 50 \%)$ decrease in apoptotic nuclear morphological change $(P<0.05)$.

Similar results were obtained when $\mathrm{H} 9 \mathrm{c} 2$ cells were subjected to isolated anoxic injury for 12 hours (Figure $2 \mathrm{~b}$ ) because EPO-mediated protection during hypoxia resulted in a mitigation of nuclear morphologic change as opposed to $\mathrm{H} 9 \mathrm{c} 2$ cells exposed to anoxia alone $(37.7 \% \pm 3.9 \%$ versus $56.8 \% \pm 6.7 \% ; P<0.01)$. Akt inhibition by the addition of the PI3K inhibitor wortmanin reversed EPO-mediated protection $(54.2 \% \pm 5.6 \%)$ after hypoxia, while ERK inhibitor (PD98059) did not (Figure 2b). Importantly, wortmanin or PD98059 compound did not significantly alter survival of $\mathrm{H} 9 \mathrm{c} 2$ cells in the absence of EPO. Figure 2, $\mathrm{c}$ and d, contains representative cellular staining with Hoechst 33258 dye. Untreated H9c2 cells exposed to ischemic injury show more positive-stained apoptotic nuclei (Figure 2c) compared with H9c2 cells treated with EPO (Figure 2d).

Effects of systemic EPO administration on in vivo cardiac function following MI. To examine potential cardioprotective effects of EPO in vivo, we chose a rabbit model of MI caused by LCx ligation (22). This model generally produces $\mathrm{LV}$ infarcts of greater than $25 \%$ and results in significant LV dysfunction both acutely and chronically (22). In these experiments, 5,000 U/kg of human recombinant EPO was randomly and blindly administered at the time of LCx ligation. Control MI rabbits received saline; we also included a sham control group with no MI and no EPO. Blood was drawn for determination of hematocrit at the time of MI (day 0 ) and daily thereafter. At day 3 after MI, we measured in vivo cardiac function by Millar catheterization. Parameters were studied both at baseline and in response to the $\beta$-adrenergic receptor ( $\beta \mathrm{AR}$ ) agonist ISO and included peak LV pressure, LV end-diastolic pressure (LVEDP), heart rate, and $\mathrm{LV}+d P / d t_{\max }$ and $\mathrm{LV}-d P / d t_{\min }$ as measures of global cardiac contractility and relaxation, respectively. Complete baseline hemodynamic measurements are shown in Table 1, and cardiac contractility and relaxation in response to $\beta A R$ stimulation are shown in Figure 3, a and b, respectively. Overall, MI rabbits had impaired LV function compared with sham controls, as we have documented previously (22), and although EPO did not completely restore global cardiac function, there was significant improvement of
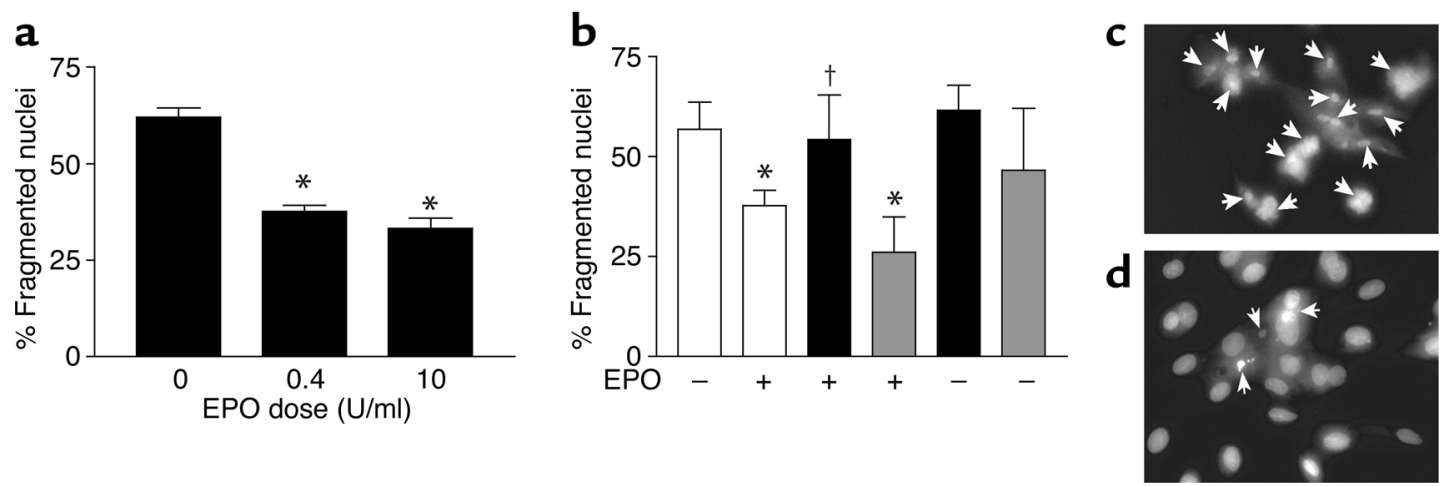

\section{Figure 2}

Apoptotic cell death in $\mathrm{H} 9 \mathrm{c} 2$ myoblasts exposed to oxidative stress and hypoxia. (a) The ratio of apoptotic cells to total adherent cells in the dish following oxidative stress $\left(\mathrm{H}_{2} \mathrm{O}_{2}\right.$ exposure). Cells were treated with $\mathrm{H}_{2} \mathrm{O}_{2}(200 \mu \mathrm{M})$ following the treatment with EPO $(0.4$ or 10 $\mathrm{U} / \mathrm{ml}, n=8$ each). Cells were stained with Hoechst 33258 dye, and nuclear morphology was revealed by fluorescent microscopy (described in Methods). Data shown are the mean \pm SEM. ${ }^{*} P<0.05$ versus untreated cells. (b) The ratio of apoptotic cells to total adherent cells in the dish after hypoxic injury. Cells were exposed to anoxia (12 hours) and percentage of nuclear fragmentation quantified under the following conditions: vehicle (control), DMSO $(n=4)$, white bars; wortmanin $(n=4)$, black bars; and PD98059 $(n=4)$, gray bars. Presence $(8 \mathrm{U} / \mathrm{ml})$ or absence of EPO is indicated by + and -, respectively. Cells were stained as above. ${ }^{*} P<0.05$ versus vehicle-treated (DMSO), ${ }^{\dagger} P<0.05$ versus EPO alone. (c and d) Representative sample of $\mathrm{H} 9 \mathrm{c} 2$ cells treated with $\mathrm{H}_{2} \mathrm{O}_{2}(200 \mu \mathrm{M})$ without EPO pretreatment (c) or with EPO (10 $\mathrm{U} / \mathrm{ml}$ ) for 24 hours (d). Arrows indicate fragmented nuclei under both conditions. 
Table 1

Summary of in vivo baseline hemodynamic data in rabbits

\begin{tabular}{lccc}
\hline & Normal $(n=5)$ & MI + saline $(n=11)$ & MI + EPO $(n=12)$ \\
Parameter & $1.97 \pm 0.25$ & $3.07 \pm 0.42^{\mathrm{A}}$ & $4.45 \pm 0.91^{\mathrm{A}}$ \\
LVEDP $(\mathrm{mmHg})$ & $72.30 \pm 1.30$ & $59.15 \pm 1.88^{\mathrm{A}}$ & $66.86 \pm 3.02^{\mathrm{A}, \mathrm{B}}$ \\
Peak LVP $(\mathrm{mmHg})$ & $2,708.96 \pm 72.58$ & $2,139.12 \pm 109.82^{\mathrm{A}}$ & $2,274.04 \pm 117.49^{\mathrm{A}}$ \\
$d P / d t_{\max }(\mathrm{mmHg} / \mathrm{s})$ & $-1,622.32 \pm 88.74^{\mathrm{A}}$ & $-1,864.79 \pm 83.30^{\mathrm{A}, \mathrm{B}}$ \\
$d P / d t_{\min }-(\mathrm{mmHg} / \mathrm{s})$ & $-2,335.14 \pm 58.56$ & $264.29 \pm 7.23^{\mathrm{A}}$ & $261.08 \pm 13.86^{\mathrm{A}}$
\end{tabular}

${ }^{A} P<0.05 \mathrm{MI}+$ saline and $\mathrm{MI}+\mathrm{EPO}$ vs. normal; ${ }^{\mathrm{B} P}<0.05 \mathrm{MI}+\mathrm{EPO}$ vs. $\mathrm{MI}+$ saline. $\mathrm{LVP}, \mathrm{LV}$ pressure; BPM, beats per minute.

inotropic reserve with EPO. Importantly, both heart rate (Figure 3c) and LVEDP (Figure 3d), which can influence global cardiac function, were not altered in either MI group. Thus, these data demonstrate a true enhancement of global cardiac function by EPO at this acute window of measurement.

In regard to these positive functional effects of $\mathrm{EPO}$ in the post-MI heart, increased $\mathrm{O}_{2}$ carrying capacity due to a higher hematocrit needs to be considered, especially since previous studies have described the beneficial effects of hematocrit augmentation on cardiac function, including the use of EPO $(32,33)$. Importantly, no discernable difference in hematocrit in rabbits was noted until day 4 after MI (Figure 3), and all post-MI physiology data were obtained on day 3 after MI when hematocrits in the two groups were effectively identical. Another confounding variable in the physiological data after MI is infarct size, since smaller infarcts would naturally have less dysfunction. Rabbit hearts were harvested on day 4 after MI and stained with TTC (see Methods). Cross sections were captured as digital photographs and analyzed by computerized planimetry (22). Saline-treated MI rabbits exhibited an average infarct of $24.0 \% \pm 3.1 \%$ of the LV free wall, while EPOtreated MI rabbits exhibited infarctions of $18.5 \% \pm 2.7 \%$ of the LV. While not statistically significant $(P=0.1)$, there appears to be a strong trend for EPO to reduce infarct size. This data prompted us to examine more closely the ischemic area of risk in these infarcted rabbit hearts (see below). Effect of EPO on $L V$ area at risk after $M I$ in rabbits. We wanted to investigate further the apparent smaller infarct size induced by EPO by examining the total area of the LV at risk of ischemic injury following MI. In this protocol, rabbits underwent a 30-minute occlusion of the LCx followed by 3 days of reperfusion. Rabbits were randomly and blindly treated with EPO or saline at the time of reperfusion as described above, and 3 days later the $L V$ ischemic area and infarcts were analyzed by standard TTC-pthalo blue staining (23, 24). Notably, while the potential ischemic zone was similar in both MI groups (EPO: $32.1 \% \pm 3.9 \%, n=5$; saline: $31.1 \% \pm 4.0 \%, n=5$ ), the percentage of infarcted tissue in this area at risk was significantly reduced in the EPO group $(13.8 \% \pm 4.7 \%)$ as compared with the saline group $(35.1 \% \pm 4.3 \%, P=0.004)$ (Figure $5 a)$. Representative staining of area at risk and infarct size can be seen in Figure 5, b and c, for saline- and EPO-treated MI hearts, respectively, demonstrating significantly less infarction (nonstained tissue) after EPO treatment.

TUNEL. Since EPO-treated rabbits have smaller infarcts as a percentage of the $\mathrm{LV}$ ischemic area at risk and thus more viable myocardium - accounting for the enhanced post-MI function - we directly assessed whether EPO is protecting ischemic myocardium against apoptosis. Specifically, we carried out TUNEL labeling of LV sections 6 hours after MI in rabbits that were treated with either EPO or saline 24 hours previously. Six hours after LCx occlusion, TUNEL-positive

\section{Figure 3}

In vivo cardiac physiology in post-MI rabbits. Hemodynamic measurements at post-MI day 3 in the experimental groups $\mathrm{MI}+$ saline $(n=11)$, filled diamonds; MI + EPO $(n=12)$, filled squares; and normal sham rabbits $(n=5)$, filled triangles. Measurements were taken at baseline (0) (see Table 1) and after progressive ISO stimulation. Data is the mean \pm SEM. ${ }^{*} P<0.05$ versus $\mathrm{Ml}+$ saline; $\uparrow P<0.05$ versus sham (ANOVA). (a) Global cardiac function measured by LV $d P / d t_{\max }$. (b) LV relaxation as measured by LV $d P / d t_{\text {min. }}$ (c) Heart rate. (d) LVEDP (EDP).
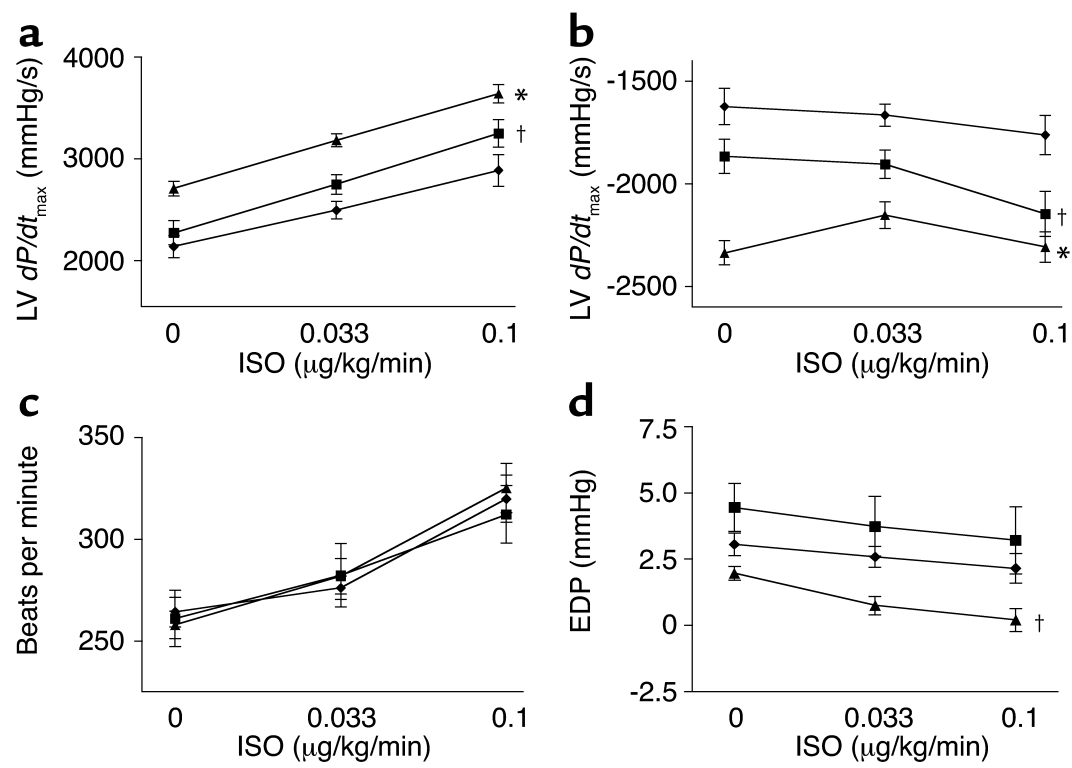


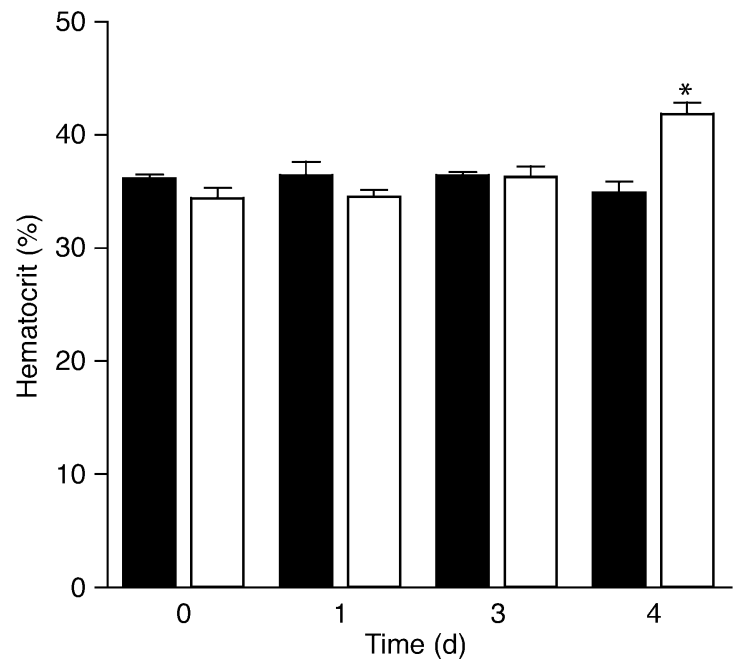

Figure 4

Hematocrit values starting at day $0(\mathrm{MI})$ and post-MI days 1,3 , and 4 in rabbits treated with EPO (MI + EPO, white bars, $n=12)$ or saline $(\mathrm{MI}+$ saline, black bars, $n=11)$. Data are the mean \pm SEM. ${ }^{*} P<0.05$ versus $\mathrm{MI}+$ saline.

cells were noted in both the saline- and EPO-treated rabbits, although significantly less staining was noted in the EPO group $(13.8 \% \pm 2.0 \%$ versus $29.3 \% \pm 3.3 \%$; $P<0.001$ ) (Figure 6a). No TUNEL-positive cells were seen in sham (no MI, no EPO) animals. Representative TUNEL-stained sections are shown in Figure 6, b and $c$, demonstrating fewer apoptotic positive cells in the EPO-treated MI rabbit heart (Figure 6c), which is consistent with lower apoptosis in cardiac myoblasts in vitro following oxidative stress and hypoxia.

In vivo myocardial Akt and ERK activation induced by $E P O$. Since the PI3K/Akt pathway as well as the ERK pathway was shown to be activated by EPO in $\mathrm{H} 9 \mathrm{c} 2$ cells and implicated in protection against hypoxic injury and perhaps mitogenesis, we examined whether these critical kinase cascades could be activated by EPO in the intact adult rabbit heart. Normal rabbits were treated with either saline (controls) or EPO $(1,000$ $\mathrm{U} / \mathrm{kg}$ ) and sacrificed 12 hours later. Phosphorylated (i.e., activated) Akt and ERK were then measured in lysates prepared from control and EPO-treated rabbit hearts by protein immunoblotting as carried out above in H9c2 cells (Figure 7). As shown in Figure 7, both Akt and ERK were found to be significantly activated in vivo in the rabbit $L V$ after $E P O$ administration. Akt was activated a modest $50 \%$ over control values (Figure 7a), while ERK activity was robustly stimulated at more than sevenfold over controls (Figure 7b). The activation of the cell survival kinase Akt by EPO treatment in the adult rabbit heart in vivo is consistent with lower apoptosis seen when EPO is given at the time of MI (Figure 6).

\section{Discussion}

Our study reveals that EPO treatment can protect the ischemic and infarcted heart by inhibition of apoptosis. This novel cardioprotective effect of EPO appears to limit infarct size by preserving myocardium in the ischemic zone, leading to enhanced cardiac contractile function and increasing inotropic reserve. This protection is seen in the absence of a change in red blood cell counts. Thus, our data support a new paradigm for EPO in the ischemic heart: protective effects on myocytes can be divorced from hematopoietic effects that may improve the supply of $\mathrm{O}_{2}$, yet predispose to thrombotic events and higher mortality. Low-dose EPO may therefore represent a novel therapeutic approach to limit myocardial injury following ischemic events.

Tissue hypoxia and inflammation has been shown to lead to $\mathrm{H}_{2} \mathrm{O}_{2}$ production secondary to respiratory uncoupling $(19,31)$. In an in vitro assessment of EPO's protective effects in cardiac cells, the heart-derived myoblast cell line, $\mathrm{H} 9 \mathrm{c} 2$, had increased cell survival upon $\mathrm{H}_{2} \mathrm{O}_{2}$ exposure as well as hypoxia. Thus, in two different in vitro model systems that can simulate in vivo conditions after ischemic insults to the heart, EPO can have direct effects to preserve cardiac cell survival. Consistent with the hypothesis that EPO can directly
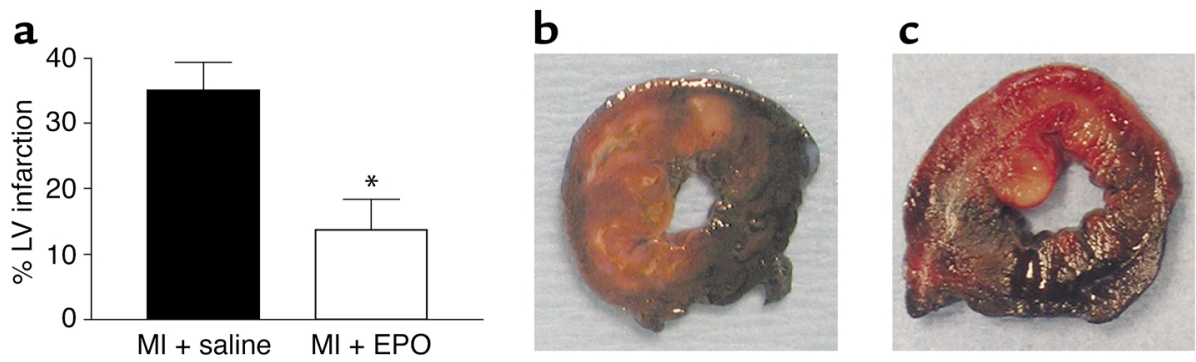

\section{Figure 5}

LV area at risk quantification after ischemia/reperfusion. (a) Graphic representation of the LV infarction size measured as the percentage of infarct of total ischemic area at risk in $\mathrm{MI}+$ saline (control) rabbits (black bar, $n=5$ ) and $\mathrm{MI}+\mathrm{EPO}$ rabbits (white bar, $n=5$ ). The LV area at risk was virtually identical in both groups (see Results). Data are the mean \pm SEM. ${ }^{*} P<0.005$ versus $\mathrm{MI}+$ saline. (b) Representative LV cross sections from a $\mathrm{MI}+$ saline (control) rabbit and (c) from a MI + EPO rabbit. The dark blue-stained areas are nonischemic tissue, and red-stained areas represent the ischemic LV area at risk. Infarcted areas are indicated by blanched (yellow-white) areas within this area at risk, with much less seen in the MI + EPO heart (c). 


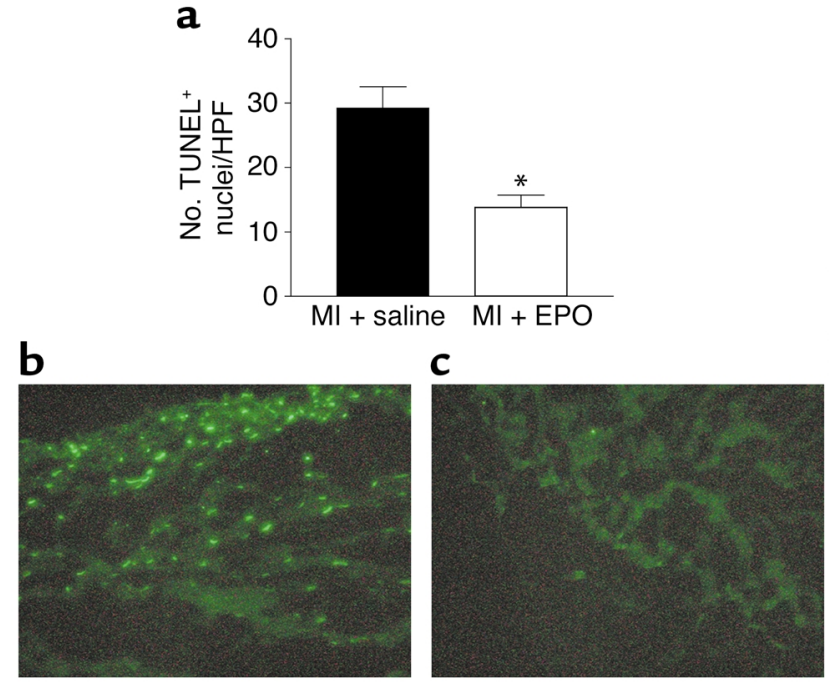

Figure 6

TUNEL staining and quantification of apoptosis in vivo in postischemic rabbit hearts. (a) Graphic representation of TUNEL-positive nuclei in $\mathrm{MI}+$ saline (control) rabbit hearts (black bar, $n=4$ ) and $\mathrm{MI}+\mathrm{EPO}$ rabbits (white bar, $n=4$ ). Data shown are the mean \pm SEM of the number of TUNEL-positive cells (nuclei) per high-power field analyzed by microscopy. ${ }^{*} P<0.05$ versus $\mathrm{MI}+$ saline (control) rabbits. (b) Representative TUNEL-stained LV section from an $\mathrm{MI}+$ saline (control) rabbit showing several positive-stained apoptotic nuclei. (c) Representative TUNEL-stained LV section from a MI + EPO rabbit with very few apoptotic nuclei.

protect cardiac cells, the EPO-R is expressed in endothelial cells (3) and cardiac tissue (4). Our own Western blot analysis confirms a strong signal for the EPO-R in rabbit whole-heart homogenate (data not shown). Importantly, both EPO production and EPOR expression (34-36) are stimulated by hypoxia (hypoxia-inducible factor) and by ROS. Future studies are needed to confirm whether this endogenous mechanism of hypoxia-induced EPO and EPO-R upregulation is indeed active in the ischemic heart in vivo to protect against programmed cell death.

The in vivo benefits of EPO extend beyond effects on ischemia because we found significant augmentation of cardiac contractility and relaxation, even when EPO was given at the time of MI. This enhanced function appears to result from less apoptosis in the ischemic area of the LV and was found not only after MI but also in an ischemia/reperfusion model. In fact, in experiments that induced the same degree of the ischemic area at risk in the LV, EPO given at the time of reperfusion significantly reduced the infarct size within this ischemic area. By decreasing infarct size, EPO preserves viable myocardium in the ischemic ventricle, resulting in improved cardiac contractile function, especially in response to the $\beta A R$ agonist ISO. $\beta A R$ density and signaling were similar in noninfarcted LV tissue from control and EPO-treated hearts (data not shown) and thus the increased inotropic reserve appears to be due simply to increased healthy myocardium. Physiological improvement after MI was seen without a change in hematocrit, so the effects cannot be attributed to improvements in $\mathrm{O}_{2}$ delivery.

The current accumulated data on the cardiovascular benefits of EPO are conflicting. On the one hand, anemia has been associated with higher mortality in cardiac patients and, by stimulating hematopoiesis, EPO may increase $\mathrm{O}_{2}$ delivery to the myocardium, resulting in some functional benefit $(24,29)$. On the other hand, correction of hematocrit may not translate into therapeutic gains. EPO administration in humans can result in hypertension (37-39) and a thrombotic diathesis (40) and may even increase mortality rates in patients with ischemic heart disease (12). In mice, EPO-mediated increases in hematocrit deplete NO and thereby predispose them to severe cardiac dysfunction $(8,41)$. Our goal, therefore, was to determine whether the effects of EPO on myocytes in vivo could be divorced from effects on hematopoietic cells. These studies demonstrate that a single dose of EPO $(1,000-5,000 \mathrm{U} / \mathrm{kg})$ administered around the time of $\mathrm{MI}$ or reperfusion can have a profound therapeutic effect independent of hematocrit. A single-dose effect of EPO also had protective effects against ischemic injury in neuronal models $(3,14)$. Thus, we anticipate that cellular protection by EPO in ischemia will be a generalizable phenomenon

In erythrocytes and neuronal cells, EPO and EPO-R stimulation can activate protein kinase cascades (15, $42,43)$. The primary kinase signaling pathway is the stress-responsive Jak2, which can lead to phosphorylation and activation of several downstream targets, including the transcription factor STAT5 (42). JakSTAT signaling is involved in prevention of neuronal apoptosis (15) and interestingly, the Jak-STAT pathway has recently been shown to play a role in cardiac ischemic preconditioning, thereby reducing ischemic damage to the heart (44). Moreover, Jak-STAT activation has been shown to prevent apoptosis in cultured myocytes (45). Our signaling data demonstrate that in cardiac cells Jak1-STAT3 is the active pathway and also that ERK MAPK and Akt (through PI3K) are robustly a

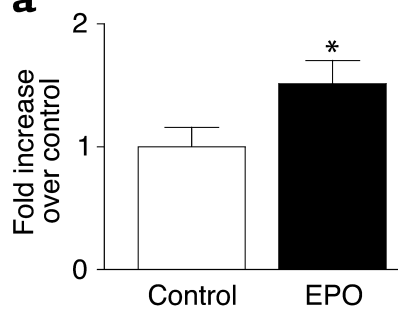

b

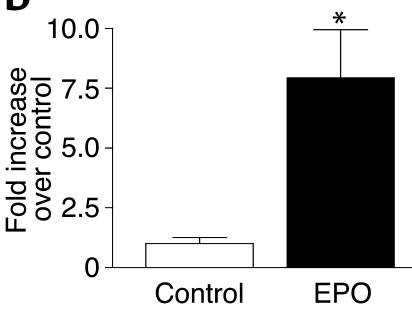

\section{Figure 7}

In vivo activation of signaling kinases in the intact adult rabbit heart after EPO treatment. Shown in graphic representation is the activation of (a) Akt and (b) ERK assessed in LV lysates prepared 12 hours after the administration of EPO $(1,000 \mathrm{U} / \mathrm{kg})$ or saline (Control). Activated Akt and ERK were assessed using anti-phospho Ab's, and activated forms were normalized to total protein content of the corresponding molecule as in Figure 1. ${ }^{*} P<0.05$ versus control values $(n=4$ each). 
stimulated by EPO treatment; thus, they appear to be important mechanistic signaling intermediates in the direct actions of EPO in cardiocytes. Results in H9c2 cells exposed to hypoxia indicate that Akt activity contributes to cardiac cell survival afforded by EPO, and, importantly, Akt activation was also seen in the intact adult rabbit heart in vivo following EPO administration. Thus, this kinase involved in cell survival signaling may be part of the pharmacologic preconditioning effects of EPO to protect the ischemic heart.

$\mathrm{EPO}$ also stimulated cellular proliferation in $\mathrm{H} 9 \mathrm{c} 2$ cells (possibly via ERK activation), which could also represent a potential contributing mechanism. Furthermore, robust ERK activation was also seen in the intact rabbit heart following EPO administration, implicating this kinase pathway in the in vivo effects of EPO. Understanding the mechanisms of signaling pathways that are involved in protection of cardiac cells from ischemic injury and death may lead to advances in the clinical management of patients with syndromes of acute ischemic injury. Collectively, our signaling data suggest likely mechanisms by which EPO protects against myocardial ischemia, although future studies will be needed to confirm these concepts.

In summary, EPO can reduce infarct size and mitigate ventricular dysfunction after MI without changing hematocrit. Improved postischemic functional recovery with EPO administration may be associated with reduced infarct size and cardiomyocyte apoptosis. Thus, a window of therapeutic opportunity may exist where a single dose of EPO following an MI or ischemic cardiac event (such as cardiac surgery or acute coronary syndromes) can offer acute protection as well as long-lasting benefit through preservation of viable myocardium during the ischemia/reperfusion period. The in vivo mechanism appears to be decreased myocyte apoptosis. Since current therapeutic strategies for ischemic heart disease are aimed at relieving the ischemia (i.e., opening of blocked arteries), EPO, by directly protecting the heart and preserving ventricular function, may represent a novel modality for the treatment of myocardial ischemia, reperfusion injury, and infarction.

\section{Acknowledgments}

The authors thank Elaine Parker, George Quick, and Andrea Koch for technical assistance and Damien Craig for technical and statistical assistance. This study was supported in part by NIH grants R01-HL59533 and R01-HL56205 (to W.J. Koch), R01-ES09206 (to J.S. Stamler), and an NIH/National Research Service Award Fellowship F32-HL70505 (to C.J. Parsa). J.S. Stamler is an Investigator of the Howard Hughes Medical Institute.

1. Gottlieb, R.A., and Engler, R.L. 1999. Apoptosis in myocardial ischemiareperfusion. Ann. N. Y. Acad. Sci. 874:412-426.

2. Siren, A.L., et al. 2001. Erythropoietin prevents neuronal apoptosis after cerebral ischemia and metabolic stress. Proc. Natl. Acad. Sci. U. S. A. 98:4044-4049.

3. Brines, M.L., et al. 2000. Erythropoietin crosses the blood-brain barrier to protect against experimental brain injury. Proc. Natl. Acad. Sci. U. S. A. 97:10526-10531.
4. Paffett-Lugassy, N., and Zon, L.I. 2002. Epo-EpoR signaling not required for cardiovascular or neural development. Blood. 100:2277. (Abstr.)

5. Erslev, A.J., and Caro, J. 1989. Erythropoietin: from mountain top to bedside. Adv. Exp. Med. Biol. 271:1-7.

6. Hayashi, T., et al. 2000. Cardiovascular effect of normalizing the hematocrit level during erythropoietin therapy in predialysis patients with chronic renal failure. Am. J. Kidney. Dis. 35:250-256.

7.Jelkmann, W. 1994. Biology of erythropoietin. Clin. Investig. 72(Suppl. 6):S3-S10.

8. Ruschitzka, F.T., et al. 2000. Nitric oxide prevents cardiovascular disease and determines survival in polyglobulic mice overexpressing erythropoietin. Proc. Natl. Acad. Sci. U. S. A. 97:11609-11613.

9. McMahon, T.J., et al. 2002. Nitric oxide in the human respiratory cycle. Nat. Med. 8:711-717.

10. Rao, S.V., and Stamler, J.S. 2002. Eythropoietin, anemia, and orthostatic hypotension: the evidence mounts. Clin. Auton. Res. 12:141-143.

11. Hebert, P.C., et al. 1999. A multicenter, randomized, controlled clinical trial of transfusion requirements in critical care. N. Engl. J. Med. 340:409-417.

12. Besarab, A., et al. 1998. The effects of normal as compared with low hematocrit values in patients with cardiac disease who are receiving hemodialysis and epoetin. N. Engl. J. Med. 339:584-590.

13. Grimm, C., et al. 2002. HIF-1-induced erythropoietin in the hypoxic retina protects against light-induced retinal degeneration. Nat. Med. 8:718-724.

14. Celik, M., et al. 2002. Erythropoietin prevents motor neuron apoptosis and neurologic disability in experimental spinal cord ischemic injury. Proc. Natl. Acad. Sci. U. S. A. 99:2258-2263.

15. Digicaylioglu, M., and Lipton, S.A. 2001. Erythropoietin-mediated neuroprotection involves cross-talk between Jak2 and NF-kappaB signalling cascades. Nature. 412:641-647.

16. Ihle, J.N. 1995. Cytokine receptor signalling. Nature. 377:591-594

17. Wojchowski, D.M., Gregory, R.C., Miller, C.P., Pandit, A.K., and Pircher, T.J. 1999. Signal transduction in the erythropoietin receptor system. Exp. Cell Res. 253:143-156.

18. Palmer, L.A., Gaston, B., and Johns, R.A. 2000. Normoxic stabilization of hypoxia-inducible factor-1 expression and activity: redox-dependent effect of nitrogen oxides. Mol. Pharmacol. 58:1197-1203.

19. Vanden Hoek, T.L., Becker, L.B., Shao, Z., Li, C., and Schumacker, P.T. 1998. Reactive oxygen species released from mitochondria during brief hypoxia induce preconditioning in cardiomyocytes. J. Biol. Chem. 273:18092-18098.

20. Kimes, B.W., and Brandt, B.L. 1976. Properties of a clonal muscle cell line from rat heart. Exp. Cell. Res. 98:367-381.

21. Foglieni, C., Meoni, C., and Davalli, A.M. 2001. Fluorescent dyes for cell viability: an application on prefixed conditions. Histochem. Cell. Biol. 115:223-229.

22. White, D.C., et al. 2000. Preservation of myocardial $\beta$-adrenergic receptor signaling delays the development of heart failure after myocardial infarction. Proc. Natl. Acad. Sci. U. S. A. 97:5428-5433.

23. Ytrehus, K., et al. 1994. Rat and rabbit heart infarction: effects of anesthesia, perfusate, risk zone, and method of infarct sizing. Am. J. Physiol. 267: $\mathrm{H} 2383-\mathrm{H} 2390$

24. Takano, H., et al. 1998. Nitric oxide synthase is the mediator of late preconditioning against myocardial infarction in conscious rabbits. Circulation. 98:441-449.

25. Mori, M., et al. 2003. Activation of extracellular signal-regulated kinases ERK1 and ERK2 induces Bcl-xL up-regulation via inhibition of caspase activities in erythropoietin signaling. J. Cell Physiol. 195:290-297.

26. Bouscary, D., et al. 2003. Critical role for PI 3-kinase in the control of erythropoietin-induced erythroid progenitor proliferation. Blood. 101:3436-3443.

27. Ratajczak, J., et al. 2001. Biological significance of MAPK, AKT and JAKSTAT protein activation by various erythropoietic factors in normal human early erythroid cells. Br. J. Haematol. 115:195-204.

28. Chong, Z.Z., Kang, J.Q., and Maiese, K. 2002. Erythropoietin is a novel vascular protectant through activation of Akt1 and mitochondrial modulation of cysteine proteases. Circulation. 106:2973-2979.

29. Neuss, M., et al. 2001. The apoptotic regulatory protein ARC (apoptosis repressor with caspase recruitment domain) prevents oxidant stressmediated cell death by preserving mitochondrial function. J. Biol. Chem. 276:33915-33922.

30. Zweier, J.L., Flaherty, J.T., and Weisfeldt, M.L. 1987. Direct measurement of free radical generation following reperfusion of ischemic myocardium. Proc. Natl. Acad. Sci. U. S. A. 84:1404-1407.

31. Chen, H.W., Chien, C.T., Yu, S.L., Lee, Y.T., and Chen, W.J. 2002. Cyclosporine A regulate oxidative stress-induced apoptosis in cardiomyocytes: mechanisms via ROS generation, iNOS and Hsp70. Br.J. Pharmacol. 137:771-781.

32. Silverberg, D.S., et al. 2000. The use of subcutaneous erythropoietin and intravenous iron for the treatment of the anemia of severe, resistant 
congestive heart failure improves cardiac and renal function and functional cardiac class, and markedly reduces hospitalizations. J. Am. Coll. Cardiol. 35:1737-1744.

33. Silverberg, D.S., et al. 2001. The effect of correction of mild anemia in severe, resistant congestive heart failure using subcutaneous erythropoietin and intravenous iron: a randomized controlled study. J. Am. Coll. Cardiol. 37:1775-1780.

34. Chandel, N.S., et al. 1998. Mitochondrial reactive oxygen species trigger hypoxia-induced transcription. Proc. Natl. Acad. Sci. U. S. A 95:11715-11720.

35. Kim, C.H., Cho, Y.S., Chun, Y.S., Park, J.W., and Kim, M.S. 2002. Early expression of myocardial HIF- $1 \alpha$ in response to mechanical stresses: regulation by stretch-activated channels and the phosphatidylinositol 3-kinase signaling pathway. Circ. Res. 90:E25-E33.

36. Shyu, K.G., et al. 2002. Intramyocardial injection of naked DNA encoding HIF-1alpha/VP16 hybrid to enhance angiogenesis in an acute myocardial infarction model in the rat. Cardiovasc. Res. 54:576-583.

37. The US Recombinant Human Erythropoietin Predialysis Study Group. 1991. Double-blind, placebo-controlled study of the therapeutic use of recombinant human erythropoietin for anemia associated with chronic renal failure in predialysis patients. Am. J. Kidney Dis. 18:50-59.
38. Lim, V.S. 1991. Recombinant human erythropoietin in predialysis patients. Am. J. Kidney Dis. 18:34-37.

39. Varet, B., Casadevall, N., Lacombe, C., and Nayeaux, P. 1990. Erythropoietin: physiology and clinical experience. Semin. Hematol. 27:25-31.

40. 1989. Polycythaemia due to hypoxaemia: advantage or disadvantage? Lancet. 2:20-22.

41. Wagner, K.F., et al. 2001. Chronic inborn erythrocytosis leads to cardiac dysfunction and premature death in mice overexpressing erythropoietin. Blood. 97:536-542.

42. Boer, A.K., Drayer, A.L., Rui, H., and Vellenga, E. 2002. ProstaglandinE2 enhances EPO-mediated STAT5 transcriptional activity by serine phosphorylation of CREB. Blood. 100:467-473.

43. Dolznig, H., et al. 2002. Apoptosis protection by the epo target bcl-x(l) allows factor-independent differentiation of primary erythroblasts. Curr. Biol. 12:1076-1085.

44. Xuan, Y.T., Guo, Y., Han, H., Zhu, Y., and Bolli, R. 2001. An essential role of the JAK-STAT pathway in ischemic preconditioning. Proc. Natl. Acad. Sci. U. S. A. 98:9050-9055.

45. Stephanou, A., et al. 2000. Ischemia-induced STAT-1 expression and activation play a critical role in cardiomyocyte apoptosis. J. Biol. Chem. 275:10002-10008. 